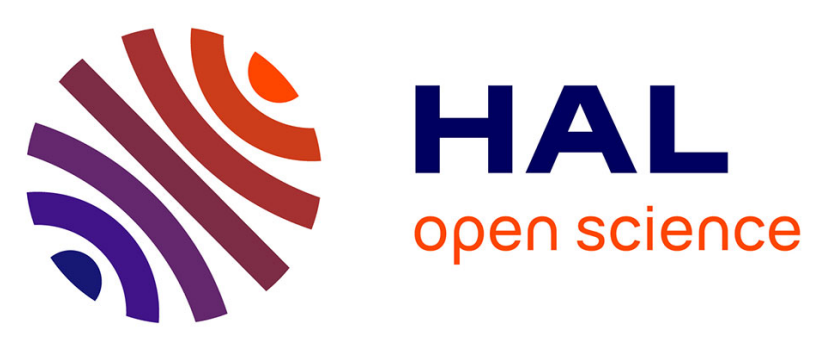

\title{
Time-varying MPC-based energy management for HEV including engine stop \& start
}

\author{
Nicoleta Stroe, Sorin Olaru, Guillaume Colin, Karim Ben-Cherif, Yann
}

Chamaillard

\section{- To cite this version:}

Nicoleta Stroe, Sorin Olaru, Guillaume Colin, Karim Ben-Cherif, Yann Chamaillard. Time-varying MPC-based energy management for HEV including engine stop \& start. 20th International Conference on System Theory, Control and Computing (ICSTCC), Oct 2016, Sinaia, Romania. pp.790 - 795, 10.1109/ICSTCC.2016.7790764 . hal-01429252

\section{HAL Id: hal-01429252 \\ https://hal-centralesupelec.archives-ouvertes.fr/hal-01429252}

Submitted on 28 Feb 2018

HAL is a multi-disciplinary open access archive for the deposit and dissemination of scientific research documents, whether they are published or not. The documents may come from teaching and research institutions in France or abroad, or from public or private research centers.
L'archive ouverte pluridisciplinaire HAL, est destinée au dépôt et à la diffusion de documents scientifiques de niveau recherche, publiés ou non, émanant des établissements d'enseignement et de recherche français ou étrangers, des laboratoires publics ou privés. 


\title{
Time-varying MPC-based energy management for HEV including engine stop \& start
}

\author{
Nicoleta Stroe*, Sorin Olaru ${ }^{\dagger}$, Guillaume Colin ${ }^{\ddagger}$, Karim Ben-Cherif* \\ and Yann Chamaillard $\ddagger$ \\ ${ }^{*}$ Renault SAS, France, ${ }^{\dagger}$ Laboratory of Signals and Systems, CentraleSupélec \\ $\ddagger$ PRISME Laboratory, Orléans University, France \\ Email: *nicoleta-alexandra.stroe@renault.com, karim.ben-cherif@ renault.com,

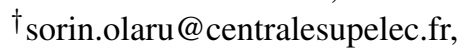 \\ ${ }^{\ddagger}$ guillaume.colin@univ-orleans.fr, yann.chamaillard@univ-orleans.fr
}

\begin{abstract}
In this paper, the energy management of hybrid electric vehicles is considered and two problems are addressed: torque split and engine stop-start policies. Model predictive control potential is proven especially for the decision to stop the engine at the proper moment and also to anticipate engine restart. A piecewise linear polynomial with respect to torque is used to approximate the fuel consumption and it will be shown that its partition can be exploited to implicitly consider engine stop decision. The strategy is applied to a dual-clutch transmission hybrid architecture which was simulated on a high-fidelity vehicle model.
\end{abstract}

\section{INTRODUCTION}

Hybrid electric vehicles (HEV) potential in minimizing fuel consumption makes them an attractive alternative to conventional vehicles. In the last decade, a considerable number of publications have enriched the research activity concerning the strategies to minimize fuel consumption and emissions or to improve drivability. From a control perspective, the problem of power split between the engine and electrical machine(s) and engine stop-start decisions are among the highest challenges for an HEV.

The literature contains already methods for calculating the power distribution and the most common are: dynamic programming, which gives the global optimum, provided the drive cycle is entirely known in advance [1], rule-based [2] and ECMS - Equivalent Consumption Minimization Strategy [3]. The latter can also be found under the form of AdaptiveECMS [4] or Telemetric-ECMS [5]. In addition, the nowadays possibility to foresee traffic conditions and the ability to integrate them in real-time algorithms, as well as the ability to handle constraints have turned the attention to Model Predictive Control (MPC) method, in its deterministic formulation [6], [7] or stochastic [8], [9].

Engine stop-start functionality did not receive the same attention in conjunction with power split problem. There are two main approaches: introduction of a binary decision variable in the optimization procedure (as in [10], where a mixedinteger quadratically constraint linear problem is formulated) or separation into a two-layer optimization. The latter is more often encountered: in [11] a constrained LP is used to compute

978-1-5090-2720-0/16/\$31.00 (c) 2016 European Union the power distribution at the top level, whereas the engine operating point optimization is performed at a bottom level; in [12] the upper level uses dynamic programming for engine ON/OFF decision and gear choice, whereas the lower level uses convex optimization to provide the torque split. The MPC benefit for stop-start strategy has been analysed in [13] for a city bus, where the energy management is also handled as a separated, low-level problem.

In this paper, the velocity profile and the gear engaged are assumed known in advance for a short time horizon. As a first contribution we show that this information will be exploited in a MPC formulation of the energy management problem, where a new optimization criterion will be introduced, with a generalized method for the calculation of the equivalence factor. A methodology to simultaneously include the torque split and engine stop-start objectives but without introducing an additional discrete variable is presented formally. The stop decision is implicitly handled by appropriate penalties on torque variations and it is taken a-posteriori, with respect to the values of computed vector of optimal commands. Finally, an additional contribution resides in the fact that a restart anticipation is introduced in order to improve the vehicle speed tracking.

The article is organized as follows: first, a powertrain control - oriented model is presented; second, the energy management problem is formulated. The simulation results obtained and the conclusions complete the paper.

\section{NOTATIONS}

- ICE - internal combustion engine

- EM - electric machine

- DCT - dual-clutch transmission

- $\mathrm{R}_{\mathrm{i}}$ - gear ratio engaged on $i^{\text {th }}$ shaft (includes neutral definition), $i \in\{1:$ odd, $2:$ even $\}$

- $\mathrm{C}_{\mathrm{i}}$ - clutch status (0 - open, 1 - closed)

- $N_{i}=\min \left(R_{i}, 1\right)$ - used to define the case where one of the shafts is decoupled

- $F D_{R_{i}}$ - axle ratio corresponding to $i$ th shaft

- $r_{i c e / e m}^{w}$ - ratio between the ICE/EM torque at wheel level and the component (ICE/ EM) torque 
- $r_{\text {at }}$ - ratio between the EM and the corresponding shaft where it is connected

- $\omega_{i c e}^{\text {ctrl }}$ - idle speed or $0 \mathrm{rpm}$, in case of engine stop

- $R_{w}$ - wheel radius

\section{POWERTRAIN CONTROL-ORIENTED MODEL}

For an HEV, the torque at the wheel level includes complete information about the system configuration, i.e. EM position and transmission type. In this paper, a DCT hybrid with EM connected to the even primary shaft (Fig. 1) will be considered, although the approach is generic for any parallel hybrid architecture, with one EM and one battery, as shown in [14]. As mentioned in the introduction, the engaged gears are pre-imposed, the variables to be determined on-line are the component torques $\left(T_{i c e}, T_{e m}\right)$, which are considered as control signals. The wheel torque is calculated from the vehicle speed $(v)$, slope $(\alpha)$ and vehicle parameters such as the mass $(m)$, aerodynamic drag coefficient $\left(c_{d}\right)$, frontal area $\left(A_{f}\right)$, rolling friction coefficients $\left(c_{r 0}, c_{r 1}\right)$ and air density $\left(\rho_{\text {air }}\right)$.

$$
\begin{aligned}
T_{w}=\left(\frac{1}{2} \rho_{a i r} A_{f} c_{d} v^{2}+\right. & \left(c_{r 1} v+c_{r 0}\right) m g \cos (\alpha) \\
& +m g \sin (\alpha)+m \dot{v}) R_{w}
\end{aligned}
$$

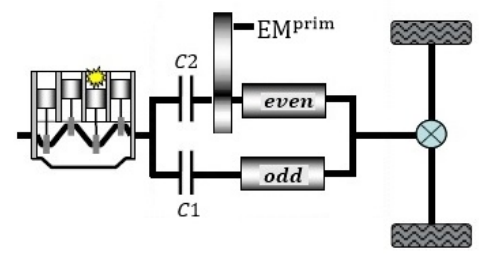

Fig. 1. DCT hybrid configuration

The shaft inertias are neglected and only the static relations for wheel torque and rotational speeds are considered, which are expressed below. The ratios $r_{i c e}^{w}, r_{e m}^{w}$ depend on the driveline states, they are therefore time-varying, which complexifies the prediction model. A description of the functional modes is given in Table I and more explanations about these expressions can be found in [14].

$T_{w}=r_{i c e}^{w} T_{i c e}+r_{e m}^{w} T_{e m}$

$r_{i c e}^{w}=F D_{\left(R_{1}\right)} R_{1} C_{1}+F D_{\left(R_{2}\right)} R_{2} C_{2}$

$r_{e m}^{w}=F D_{\left(R_{1} C_{1} C_{2}+R_{2}\right)}\left(R_{1} C_{1} C_{2}+R_{2}\right)$ rat $_{\text {em }}$

$\omega_{i c e}=r_{i c e}^{w} \frac{v}{R_{w}}+\left(1-C_{1}-N_{2} C_{2}\right) \omega_{i c e}^{c t r l}$

$\omega_{e m}=r_{e m}^{w} \frac{v}{R_{w}}+\operatorname{rat}_{e m} C_{2}\left(1-C_{1}\right)\left(1-N_{2}\right) \omega_{i c e}^{c t r l}$

These static relations can be reduced to the compact form below, if the terms in $\omega_{i c e}^{\text {ctrl }}$ are neglected:

$$
T_{w}=\left[\begin{array}{ll}
r_{i c e}^{w} & r_{e m}^{w}
\end{array}\right]\left[\begin{array}{l}
T_{i c e} \\
T_{e m}
\end{array}\right],\left[\begin{array}{l}
\omega_{i c e} \\
\omega_{e m}
\end{array}\right]=\left[\begin{array}{l}
r_{i c e}^{w} \\
r_{e m}^{w}
\end{array}\right] \frac{v}{R_{w}}
$$

TABLE I

HYBRID DCT MODES AS FUNCTIONS OF CLUTCHES STATES AND $N_{2}$

\begin{tabular}{|c|c|c|c|}
\hline$C_{1}$ & $C_{2}$ & $N_{2}$ & Case \\
\hline 0 & 0 & 0 & standstill, sailing \\
\hline 0 & 0 & 1 & electric driving, regenerative braking \\
\hline 0 & 1 & 1 & hybrid or conventional, even gear engaged \\
\hline 0 & 1 & 0 & charge during standstill \\
\hline 1 & 0 & 0 & conventional driving, odd gear engaged \\
\hline 1 & 0 & 1 & hybrid driving \\
\hline 1 & 1 & 0 & take-off, charge during driving \\
\hline
\end{tabular}

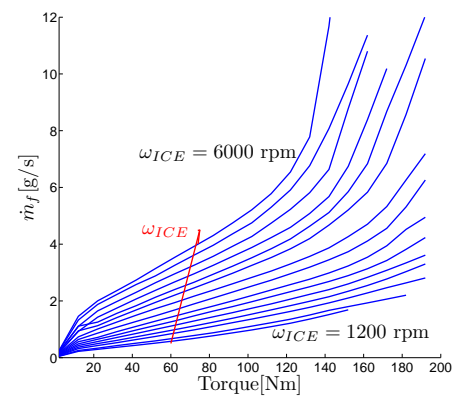

Fig. 2. Fuel consumption $[\mathrm{g} / \mathrm{s}]$ with respect to torque; curves for different rotational speed values

The only dynamical part of the system considered is the battery state-of-charge (SOC), for which an internal resistance model is used, with $O C V$ - open circuit voltage, $R$ - internal resistance, $Q_{\max }$ - battery capacity:

$$
S \dot{O} C=-\frac{O C V_{(S O C)}-\sqrt{O C V_{(S O C)}^{2}-4 R_{(S O C)} P_{b}}}{2 R_{(S O C)} Q_{\max }}
$$

with the battery power given by:

$$
P_{b}=\frac{\pi}{30} \omega_{e m} T_{e m}+\operatorname{loss}\left(\omega_{e m}, T_{e m}\right) .
$$

\section{A. Fuel consumption approximation}

The considered system is equiped with an $1.2 \mathrm{~L}$ SI engine whose fuel mass flow $\left(\dot{m}_{f}[\mathrm{~g} / \mathrm{s}]\right)$ with respect to torque is depicted in Fig. 2, for a family of rotational speeds. This dependance is extracted from a nonlinear map, defined for a torque grid with 20 values. As a contribution, in the present work we advocate the representation of the fuel consumption in terms of a piecewise first order polynomial with respect to torque, whose coefficients depend on rotational speed. For rotational speed values between two curves, an interpolation is performed:

$$
\begin{gathered}
\dot{m}_{f}=\alpha_{i}\left(\omega_{i c e}, T_{i c e}^{\min _{i}, \max _{i}}\right) T_{i c e}+\beta_{i}\left(\omega_{i c e}, T_{i c e}^{\min _{i}, \max _{i}}\right) \\
\text { for } T_{i c e}^{\text {min }_{i}} \leq T_{i c e} \leq T_{i c e}^{\max _{i}}, i=1 \ldots N_{\text {part }}
\end{gathered}
$$

For the particular case of the engine under study, a number of $N_{\text {part }}=8$ partitions was found to give accurate results. 


\section{ENERGY MANAGEMENT - PROBLEM FORMULATION}

The main goal of this work is to minimize the fuel consumption for an HEV without an external recharge, by computing the optimal torque distribution between the two energy sources. It is assumed that the wheel torque demand and the engaged gear can be accurately predicted for several seconds (around $5 \mathrm{~s}$ ). This prediction horizon value favors engine stop inclusion and thus, a higher consumption gain can be obtained, without the risk of affecting the vehicle speed tracking. First, an optimization criterion for the torque split problem will be introduced and then it will be shown how it can be modified to tackle the engine stop-start.

\section{A. Optimization criterion}

The optimization problem can be translated into a trade-off between the use of two power sources. The EM can provide alone the demanded torque or it can assist the engine, but in the absence of an external recharge, attention must be paid to battery consumption during driving. The criterion chosen in this paper incorporates this trade-off and its formulation is inspired from the ECMS design:

$$
\begin{gathered}
\min _{u_{k}} P_{f_{k}}+\lambda_{k} P_{e_{k}} \\
P_{f_{k}}=H_{L V} \dot{m}_{f_{k}}, \quad P_{e_{k}}=O C V_{k} S \dot{O} C
\end{gathered}
$$

where $k$ is the current step, $u_{k}$ is the engine torque, $H_{L V}$ is the lower heating value of the fuel and $\lambda_{k}$ is an equivalence factor. As a difference with ECMS, the squared values of the powers are used instead, in order to obtain convexity structural advantages:

$$
\min _{u} \sum_{i=k}^{k+N-1} P_{f}^{2}(i)+\lambda_{k}^{2}\left(P_{e_{k}}(i)-P_{\text {emin }_{k}}(i)\right)^{2}
$$

where $N$ is the prediction horizon, $u$ is a vector with ICE torques of length $N$ and $P_{e m i n}$ is the electrochemical power minimal value. By using the squared values of the powers, all sign information is however lost. Hence, the introduction of $P_{\text {emin }}$ aims to properly handle the sign of electrochemical power, that can be either positive or negative.

The value of $\lambda$ that assures a proper balance between the two sources is strongly dependent on the drive cycle characteristics, which cannot be entirely known in advance. Here, a generalized method calculates the weighting $\lambda$ as the ratio between the variations of the two powers:

$$
\lambda_{k, i}=-\frac{P_{f}\left(\bar{u}_{i}\right)-P_{f}\left(\underline{u}_{i}\right)}{P_{e}\left(\bar{u}_{i}\right)-P_{e}\left(\underline{u}_{i}\right)}
$$

where $\underline{u}_{i}, \bar{u}_{i}$ are the minimum and the maximum limit, respectively of engine torque at the $i$ th step as they are determined from constraints activation. As an alternative, to decrease the sensitivity with respect to the anticipatory information on the constraints activation, a constant $\lambda_{k}$ is set during the prediction, calculated as an average in a receding horizon manner. The goal is to find at each step an equivalence factor that approximates the average of future variations of the two powers:

$$
\lambda_{k}=\frac{1}{N_{\text {pred }}} \sum_{i=k}^{k+N_{\text {pred }}-1} \lambda_{k, i}
$$

where $N_{\text {pred }} \geq N$. Such an average-based formulation has been further retained for the present study. This second choice of the prediction horizon for $\lambda$ calculation relies on the lookahead possibilities, that can be greater than those exploited in the MPC: for instance, a two-minute traffic prediction is a reasonable value to define the driving style variability and it is coherent with the actual possibilities of data acquisition. In principle, this choice leads to a variation of the cost function at a slower rate than the MPC optimal control sequence. However, for the MPC optimization itself a smaller horizon is chosen for two main reasons:

- computational requirements

- to limit the impact of prediction model inaccuracies that arise as a consequence of linearization, as will be shown in the next subsection.

\section{B. Problem formulation within time-varying MPC framework}

The model-based predictive control strategy considered next involves the resolution of a finite-time horizon optimization problem, at each time instant $k$ :

$$
\begin{gathered}
\min _{U} \sum_{i=1}^{N}\left(\Delta x_{k+i}^{T} Q \Delta x_{k+i}+\Delta u_{k+i-1}^{T} R \Delta u_{k+i-1}\right) \\
\text { s.t. }\left\{\begin{array}{l}
x_{\min } \leq x_{k+i} \leq x_{\max }, \quad i=1, \ldots, N \\
u_{\min } \leq u_{k+i-1} \leq u_{\max }, \quad i=1, \ldots, N
\end{array}\right.
\end{gathered}
$$

where $\Delta x, \Delta u$ denote the state and command variations, respectively and $U$ is a compact vector integrating all the future commands along the prediction horizon. The problem is reformulated as a quadratic programming:

$$
\begin{gathered}
\min _{U} \frac{1}{2} U^{T} H U+F^{T} U \\
\text { s.t. }\left\{\begin{array}{l}
A_{\text {ineq }} U \leq b_{\text {ineq }} \\
A_{e q} U=b_{\text {eq }}
\end{array}\right.
\end{gathered}
$$

where $H$ is positive definite and all matrices are obtained using algebraic manipulations over (14). For the current optimization problem, the only state variable is SOC whose model is nonlinear as given by (8). Here, a linearization at the operating point is performed, under the assumption that $O C V$ and $R$ are constant during the prediction. A Linear Time Varying (LTV) system is thus obtained:

$$
x_{k+1}=A_{k} x_{k}+B_{k} u_{k}+D_{k}
$$

where $x=S O C, A_{k}=1, u_{k}=T_{I C E}(k), D_{k}$ is a residual term due to linearization. As mentioned in the introduction, the wheel torque $\left(T_{w}\right)$ and the rotational speeds of components $\left(\omega_{I C E}, \omega_{E M}\right)$ are known in advance. It can be noticed that $A_{k}$ is constant, which reflects the integral behaviour of SOC. The model complexity is given by the time-varying characteristic 
of $B_{k}$ and $D_{k}$, which depend on EM rotational speed and torque demand and whose expressions are detailed below:

$$
\begin{aligned}
B_{k} & =\frac{\pi}{30} Q_{\max } \frac{\omega_{e m_{k}}}{f_{k}} \frac{r_{i c e_{k}}^{w}}{r_{e m_{k}}^{w}} \Delta t_{o p t} \\
D_{k} & =\frac{-O C V+f_{k}}{2 R Q_{\max }} \Delta t_{o p t}-B_{k} u_{k-1,0}
\end{aligned}
$$

where $\Delta t_{\text {opt }}$ is the MPC sampling time and $u_{k-1,0}$ is the current engine torque. Let $T_{e m}^{k \mid k-1}=$ $\frac{1}{r_{e m_{k}}^{w}}\left(T_{w}(k)-r_{i c e_{k}}^{w} u_{k-1,0}\right)$. Then:

$f_{k}=\sqrt{O C V^{2}-4 R\left(\frac{\pi}{30} \omega_{e m_{k}} T_{e m}^{k \mid k-1}+\operatorname{loss}\left(w_{e m_{k}}, T_{e m}^{k \mid k-1}\right)\right)}$

After linear algebra manipulations, we have:

$$
\begin{aligned}
& X_{k}=\Phi_{k} x_{k}+\Psi_{k} U_{k}+V_{k} \\
& \Phi_{k}(i)=\operatorname{ones}(N, 1) \\
& \Psi_{k}(i, j)= \begin{cases}B_{k+j-1} & \text { if } j \leq i \\
0 & \text { otherwise }\end{cases} \\
& V_{k}^{T}=\left[\begin{array}{llll}
D_{k} & D_{k}+D_{k+1} & \ldots & \sum_{i=1}^{N} D_{k+i-1}
\end{array}\right]
\end{aligned}
$$

where $x_{k}$ is the SOC value at instant $k$ and $X_{k}, U_{k}$ the vectors of future states and commands, respectively.

Penalties on torque variations can also be introduced for drivability reasons. Let:

$$
\begin{aligned}
& \Delta U_{k}=D_{\Delta} U_{k}+U_{k 0} \\
& U_{k 0}^{T}=\left[\begin{array}{llll}
u_{k-1,0} & 0 & \ldots & 0
\end{array}\right] \\
& D_{\Delta}(i, j)= \begin{cases}-1 & i=j \\
1 & i=j+1 \\
0 & \text { otherwise }\end{cases}
\end{aligned}
$$

With these notations, the criterion (11) can be reformulated as (15), with:

$$
\begin{aligned}
& H_{k}=\bar{\alpha}_{k}^{2}+q_{k}^{2} \bar{B}_{k}^{2} \\
& F_{k}=\bar{\alpha}_{k} \bar{\beta}_{k}-q_{k}^{2} \bar{B}_{k} U_{k}^{\max }
\end{aligned}
$$

where $q_{k}=\lambda_{k} \frac{1}{H_{L V}} Q_{\max } O C V_{k}, \quad \bar{B}_{k}=\operatorname{diag}\left(B_{k+i-1}\right)$, $U_{k}^{\max }$ is a vector with the input upper bounds, $\bar{\beta}(i)=\beta\left(\omega_{i c e, k+i-1}, T_{i c e, k+i-1}^{\min , \max }\right)$ and $\bar{\alpha}_{k}=$ $\operatorname{diag}\left(\alpha\left(\omega_{i c e, k+i-1}, T_{i c e, k+i-1}^{\min , \max }\right)\right)$, as in (9).

If penalties on torque variations are also considered, the relations (20) are updated as below, with $R_{\Delta_{k}} \geq 0$ the matrix of penalties:

$$
\begin{aligned}
& H_{k}=\bar{\alpha}_{k}^{2}+q_{k}^{2} \bar{B}_{k}^{2}+D_{\Delta}^{T} R_{\Delta_{k}} D_{\Delta} \\
& F_{k}=\bar{\alpha}_{k} \bar{\beta}_{k}-q_{k}^{2} \bar{B}_{k} U_{k}^{\max }+D_{\Delta}^{T} R_{\Delta_{k}} U_{k 0}
\end{aligned}
$$

The ICE and the EM have physical limitations of torque and power, which are directly determined from the rotational speeds. For SOC distance-varying limits are considered, as defined by the equations below and depicted in Fig. 3:

$$
\begin{aligned}
& S O C_{k}^{\text {min }}=S O C_{0}-\left(S O C_{0}-S O C_{\text {min }}\right) e^{1-\frac{1}{1-\frac{d_{k}}{d T_{0}}}} \\
& S O C_{k}^{\text {max }}=S O C_{0}+\left(S O C_{\max }-S O C_{0}\right) e^{1-\frac{1}{1-\frac{d_{k}}{d T o t}}}
\end{aligned}
$$

where $S O C_{\min }, S O C_{\max }$ are the physical SOC limitations (here set at $20 \%$ and $90 \%$, respectively), $d_{k}$ is the current distance and $d$ Tot is the total distance.

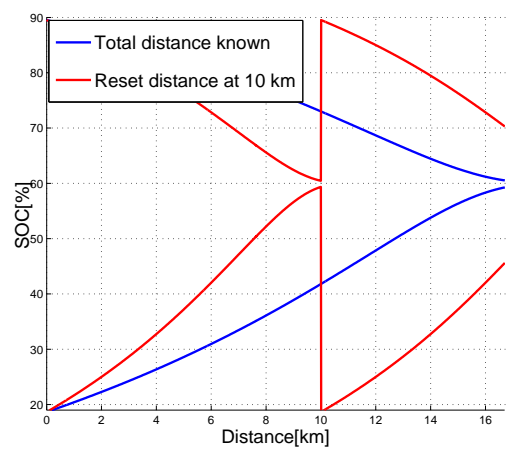

Fig. 3. SOC limits for 2 cases: total distance known and reset distance fixed at $10 \mathrm{~km}$, respectively

For an HEV without an external recharge, battery depletion must be avoided and these constraints profile on SOC enforces convergence at the end of the drive cycle toward the initial value, if the total distance is known. If this information is not available a reset distance is used instead, whose value can be determined from the driver history data. In simulation, this distance can be adjusted to either 5 or $10 \mathrm{~km}$. The constraints can therefore be expressed as:

$$
\begin{aligned}
& T_{i c e}^{\text {min }}\left(\omega_{i c e}\right) \leq T_{i c e} \leq T_{i c e}^{\max }\left(\omega_{i c e}\right) \\
& T_{e m}^{m i n}\left(\omega_{e m}\right) \leq T_{e m} \leq T_{e m}^{\max }\left(\omega_{e m}\right) \\
& P_{e m}^{m i n}\left(\omega_{e m}\right) \leq \frac{\pi}{30} \omega_{e m} T_{e m} \leq P_{e m}^{\max }\left(\omega_{e m}\right) \\
& S O C_{k}^{\text {min }}-\epsilon_{k} \leq X_{k} \leq S O C_{k}^{\max }+\epsilon_{k}
\end{aligned}
$$

where $\epsilon_{k}$ are slack variables used to relax the SOC constraints and hence, avoiding infeasibility problems which may occur especially toward the reset distance, where the range of variation is narrow.

As mentioned in section II, the fuel consumption map is partioned in several torque-dependent regions to which a first order polynomial approximation is associated. A drawback of such piecewise approximation approach is that the physical constraints are parameterized according to the partitions and thus, upon the optimization the fuel consumption parameters will evolve. The first step is to verify if a unique interval can be found:

$$
\begin{gathered}
\text { if } \exists j \text { s.t } .\left[U_{k}^{\min }(i) ; U_{k}^{\max }(i)\right] \subseteq\left[T_{i c e}^{\text {min }_{j}} ; T_{i c e}^{\max _{j}}\right] \\
\text { then } n_{p w l}(i)=j, \quad j=1 \ldots N_{\text {part }}
\end{gathered}
$$


where $n_{p w l}(i)$ denotes the region for the $i$ th command and $T_{i c e}^{\min _{j}}, T_{i c e}^{\max _{j}}$ represent the torque limits for the $j$ th region, as in (9).

If this condition is not satisfied, the choice of the consumption region relies mostly on the current engine torque value. Let $n_{0}$ be the corresponding region. Then:

$$
\begin{aligned}
\text { if } & {\left[T_{i c e}^{\min _{n}} ; T_{i c e}^{\max _{n_{0}}}\right] \cap\left[U_{k}^{\min }(i) ; U_{k}^{\max }(i)\right] \neq \emptyset } \\
& \text { then } n_{p w l}(i)=n_{0}
\end{aligned}
$$

Finally, if no intersection is found between the torque range and the current region, a neighbour interval is selected.

$$
\begin{aligned}
& n_{\text {pwl }}(i)=j, \quad j=1 \ldots N_{\text {part }} \\
& \min _{j}\left|n_{0}-j\right| \\
& \quad \text { s.t. }\left[T_{i c e}^{\text {min }} ; T_{i c e}^{\max _{j}}\right] \cap\left[U_{k}^{\text {min }}(i) ; U_{k}^{\max }(i)\right] \neq \emptyset
\end{aligned}
$$

New limits are thus imposed:

$$
\begin{aligned}
& U_{k}^{\min }(i)=\max \left(U_{k}^{\min }(i), T_{i c e}^{\min _{n_{p w l}(i)}}\right) \\
& U_{k}^{\max }(i)=\min \left(U_{k}^{\max }(i), T_{i c e}^{\text {max }_{n_{p w l}(i)}}\right)
\end{aligned}
$$

After each optimization step, a saturation check is performed for the commands whose physical limitations span several consumption regions. If the command lies within its defined limits (27), no action is taken. If saturation occurs, $n_{p w l}$ is shifted toward left or right, depending on whether the lower or the upper bound was activated, and a new optimization is launched with this update. The procedure is repeated until a chattering effect is encountered, as in (28), or a maximal number of iterations is reached.

$U_{k}^{(i t e r)}(i)= \begin{cases}U_{k}^{\text {min },(\text { iter })}(i), & n_{\text {pwl }}^{(\text {iter })}(i)=n_{\text {pwl }}^{(i t e r-1)}(i)+1 \\ U_{k}^{\max ,(\text { iter })}(i), & n_{\text {pwl }}^{(\text {iter })}(i)=n_{\text {pwl }}^{(i t e r)}(i)-1\end{cases}$

where the superscript iter denotes the iteration number.

\section{ICE stop-start inclusion}

It is assumed that the driveline states are calculated at a high level (supervisor) and based on this data, a torque split is computed at a lower level. A simple decision of ICE stop-start can be taken based only on the anticipation of idling periods, longer than the cost of a restart, which is usually expressed in seconds of idling. However, further improvement in fuel consumption can be achieved with MPC, which calculates a sequence of future commands.

Let $t_{i d l e}$ be the number of seconds of idling which translates the cost of an ICE restart, $c t_{\text {on }}$ the number of steps since an ICE restart and $c t_{\text {off }}$ the number of steps since an ICE stop. Then at step $k$ the engine is shut off for:

$$
\begin{aligned}
& U_{k}\left(1: N_{\text {stop }}\right) \leq T_{i c e}^{t h r} \\
& c t_{\text {on }}>N_{\text {start }}
\end{aligned}
$$

where $N_{\text {stop }}=\frac{t_{i d l e}}{\Delta t_{\text {opt }}}$ and $T_{i c e}^{t h r}$ is a threshold value for ICE torque below which the stop of the engine is preferable over the considered length of the prediction horizon. The condition (29b) assures that the engine is not stopped shortly after a restart decision. If the engine is already stopped, a restart is demanded if the first calculated command is superior to $T_{i c e}^{t h r}$. A $N_{o n}$ - step anticipation for ICE restart is also introduced: if the command from the position $1+N_{\text {on }}$ exceeds the threshold torque value and the engine has already been stopped for at least $N_{\text {stop }}$ steps, a restart command is activated. For the case considered, $N_{\text {on }}$ was set to 1 .

In addition, all stop conditions are subject to powertrain inhibition functions, where safety requirements are formulated (SOC level, fuel temperature, altitude value, aftertreatement etc). This stop-start strategy is described by the diagram in Fig. 4.

The PWL approximation introduced in the previous section is particularly suitable for this problem because it allows the demarcation of the low torque region, where $T_{i c e}^{t h r}$ is included: $T_{i c e}^{\min _{1}} \leq T_{i c e}^{t h r} \leq T_{i c e}^{\max _{1}}$. The first step is to identify the sequences of commands with a length of at least $N_{\text {stop }}$ for which the first region was selected for the fuel consumption approximation. Then, for these sequences, penalties on torque variations are introduced through the matrix $R_{\Delta_{k}}$, as it appears in formulation (21) of the optimization criterion:

$$
R_{\Delta_{k}}(i+1: j, i+1: j) \neq 0, \text { for }\left\{\begin{array}{l}
j-i \geq N_{\text {stop }} \\
n_{p w l}(i: j)=1
\end{array}\right.
$$

The purpose of this strategy is to force the entire sequence to either go toward zero (and the engine can therefore be stopped) or to reach the upper limit of the interval and then to commute toward higher values. The cost of an ICE restart is implicitly handled by the length of the sequence, $N_{\text {stop }}$. If the engine has been stopped for $c t_{o f f}<N_{\text {stop }}$, then penalties are introduced for the first $N_{\text {stop }}-c t_{\text {off }}$ commands, in order to maintain the calculated torque close to zero and hence, the ICE stopped:

$$
\begin{aligned}
& R_{\Delta_{k}}\left(1: N_{\text {stop }}-c t_{\text {off }}, 1: N_{\text {stop }}-c t_{\text {off }}\right) \neq 0 \\
& c t_{\text {off }}<N_{\text {stop }}
\end{aligned}
$$

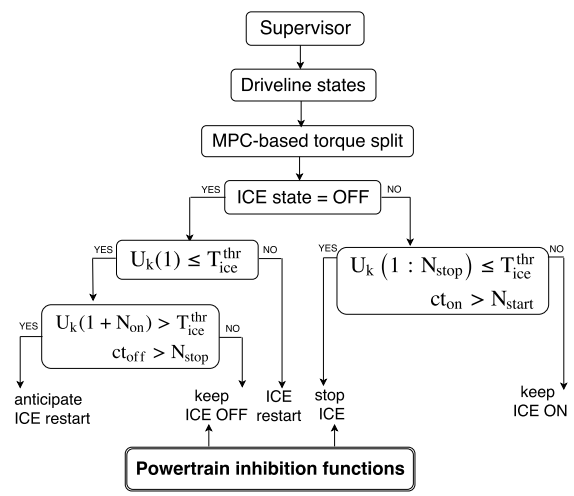

Fig. 4. ICE stop-start strategy

\section{Simulation AND RESUltS}

The proposed strategy was validated in Matlab/Simulink environment and for the vehicle dynamics a high-fidelity 
model designed in AMEsim was used. Two representative scenarios for engine stop-start are considered: Artemis urban and traffic jam, whose velocity profiles are depicted in Fig. 5. The future wheel torque demand cannot be perfectly known in advance, but for a short horizon the velocity can be accurately predicted and therefore, it is possible to obtain an accurate estimation of the wheel torque, for a given driver.
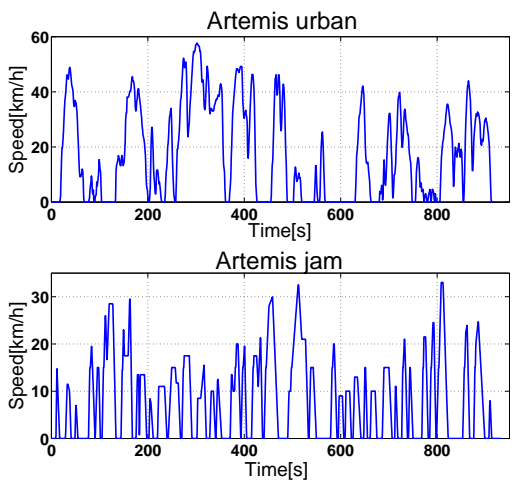

Fig. 5. Reference speed for Artemis urban and traffic jam, respectively

First, simulations were carried without the MPC- based ICE stop-start decision. For this scenario, the engine is stopped during regenerative braking and electrical traction phases (imposed for very low speeds). A comparison is made with the ECMS method, defined by (10). In [15] is shown that this Hamiltonian-based method, with a constant, drive cycle dependent adjoint state, gives close to global optimum results. Therefore, for each speed profile a constant equivalence factor was found such that the final SOC was the same as for the case with an MPC energy management, in order to make a fair comparison between the two strategies. With MPC, a degradation of only $6 \%$ from the ECMS is observed for Artemis urban and of $14 \%$ for Artemis jam, according to Table II (initial SOC fixed at $S O C_{0}=50 \%$ ).

TABLE II

FUEL CONSUMPTION [L/100KM] AND FINAL SOC[\%] FOR MPC (WITH AND WITHOUT S\&S) AND ECMS

\begin{tabular}{|c|c|c|c|}
\hline \multicolumn{2}{|c|}{ Dethod } & Artemis urban & Artemis jam \\
\hline \multicolumn{2}{|c|}{ ECMS $^{*}$} & $\begin{array}{c}6.34 \\
(53.5 \%)\end{array}$ & $\begin{array}{c}6.8 \\
(53 \%)\end{array}$ \\
\hline \multirow{3}{*}{ MPC } & $1 \mathrm{~s}$ & $\begin{array}{c}6.8 \\
(54 \%)\end{array}$ & $\begin{array}{c}7.85 \\
(54 \%)\end{array}$ \\
\cline { 2 - 4 } & \multirow{2}{*}{$5 \mathrm{~s}$} & $\begin{array}{c}6.72 \\
(53.5 \%)\end{array}$ & $\begin{array}{c}7.76 \\
(53 \%)\end{array}$ \\
\hline \multirow{2}{*}{ MPC } & $1 \mathrm{~s}$ & $\begin{array}{c}6.8 \\
(54 \%)\end{array}$ & $\begin{array}{c}7.85 \\
(54 \%)\end{array}$ \\
\cline { 2 - 4 }$(\mathrm{S} \& S)$ & $5 \mathrm{~s}$ & $\begin{array}{c}6.63 \\
(53.5 \%)\end{array}$ & $\begin{array}{c}7.4 \\
(53 \%)\end{array}$ \\
\cline { 2 - 5 } & \multirow{2}{*}{5} & &
\end{tabular}

For a constant equivalence factor $\lambda$, determined offline for each drive cycle

For the MPC-based stop-start, the threshold torque value was chosen as $T_{i c e}^{t h r}=7 \mathrm{Nm}$, the cost of a restart was set to $2 \mathrm{~s}$ of idling, $t_{\text {idle }}=2, \Delta t_{\text {opt }}=0.5 \mathrm{~s}$ and therefore, $N_{\text {stop }}=4$.
It can be seen that an increase in the prediction horizon is especially relevant for the engine stop inclusion, for $N>t_{i d l e}$. This is also depicted in Fig. 6, where it can be observed that there are more frequent stops for a prediction of $5 \mathrm{~s}$ than for $1 \mathrm{~s}$ and a fuel consumption gain up to $5 \%$ can be obtained.
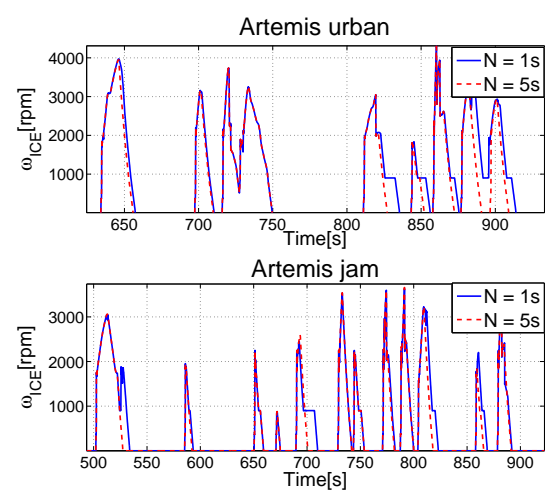

Fig. 6. MPC-based stop-start included, $\omega_{I C E}$ for two values of the prediction horizon: 1s and 5s, respectively; Artemis urban and traffic jam

\section{CONCLUSIONS AND OUTLOOK}

An MPC strategy for the energy management of an HEV has been proposed. The engine stop-start has been equally addressed, without the introduction of a binary decision variable in the optimization problem. The assumption of an accurate prediction for the wheel torque demand and the gear engaged is not restrictive due to a short prediction horizon choice. Encouraging results can be obtained with a prediction of only 5 s, notably when the engine stop is included.

An alternative solution to the engine stop-start problem, where the cost of a restart is explicitly introduced in the optimization criterion, as well as the inclusion of a terminal cost to guarantee stability can be further investigated.

\section{REFERENCES}

[1] O. Sundstrom, L. Guzzella, and P. Soltic, "Optimal hybridization in two parallel hybrid electric vehicles using dynamic programming," Proc.17th World Congress IFAC, 2008

[2] D. Goerke, M. Bargende, U. Keller, N. Ruzicka, and S. Schmiedler, "Optimal control based calibration of rule-based energy management for parallel hybrid electric vehicles," SAE Tech. Paper, vol. 2015-01-1220, 2015.

[3] A. Sciaretta, M. Back, and L. Guzzella, "Optimal control of parallel hybrid electric vehicles," IEEE Trans. Control Syst. Technol., vol. 12, No 3, 2004.

[4] C. Musardo, G. Rizzoni, Y. Guezennec, and B. Staccia, "A-ECMS an adaptive algorithm for hybrid electric vehicle energy management," European Journal of Control, 2005.

[5] A. Sciarretta, L. Guzzella, and M. Back, "A real-time optimal control strategy for parallel hybrid vehicles with on-board estimation of the control parameters," Proc. IFAC Symp. Adv. Automotive Control, 2004.

[6] S. D. Cairano, W. Liang, I. Kolmanosky, M. Kuang, and A. Phillips, "Engine power smoothing energy management strategy for a series hybrid electric vehicle," American Control Conf., 2011.

[7] Z. Lu, J. Song, H. Yuan, and L. Shen, "MPC based torque distribution strategy for energy management of power-split hybrid electric vehicles," Proc. 32nd Chinese Control Conf., July 2013.

[8] G. Ripaccioli, D. Bernardini, S. Cairano, A. Bemporad, and I. Kolmanovsky, "A stochastic MPC approach for series hybrid electric vehicle power management," American Control Conf., 2010. 
[9] M. Josevski and D. Abel, "Energy management of a parallel hybrid electric vehicles based on stochastic model predictive control," 19th World Congress, IFAC, 2014.

[10] R. Beck, A. Bollig, and D. Abel, "Comparison of two real-time predictive strategies for the optimal energy management of a hybrid electric vehicle," E-COSM - Rencontres Scientifiques de l'IFP, 2006.

[11] S. Hahn, H. Waschl, G. Steinmaurer, and L. del Re, "Extension of a linear optimal control strategy for HEV," European Control Conf., 2015.

[12] T. Nuesch, P. Elbert, M. Flankl, C. Onder, and L. Guzzella, "Convex optimization for the energy management of hybrid electric vehicles considering engine start and gearshift costs," Energies, vol. 7, 834 856,2014

[13] R. Dudek, V. Smidl, and Z. Peroutka, "Start - stop system for a city bus based on model predictive control," Industrial Electronics Society, IECON, 2014.

[14] N. Stroe, G. Colin, K. Ben-Cherif, S. Olaru, and Y. Chamaillard, "Towards a generic control-oriented model for HEV predictive energy management," 8th IFAC Int. Symp. Advances Automotive Control, 2016.

[15] L. Serrao, S. Onori, and G. Rizzoni, "A comparative analysis of energy management strategies for hybrid electric vehicles," Journal of Dynamic Systems, Measurement and Control, vol. 133, 2011. 